\title{
Aproximación psicosocial a la adultez mayor en Chile: cultura, políticas y algunos desafíos teóricos
}

\author{
Psychosocial approach to the elderly in Chile: culture, policies \\ and some theoretical challenges
}

Abordagem psicossocial da terceira idade no Chile: cultura, políticas e alguns desafios teóricos

Emilio Moyano Díaz*

\section{RESUMEN}

Se revisa literatura acerca de la adultez mayor (AM) en Chile como parte del desarrollo psicológico normal, sus características y desafíos, y contextualizada socioculturalmente. Se efectúa una caracterización sociodemográfica de este colectivo en crecimiento y se resumen algunas ideas centrales de las políticas sociales al respecto. Las personas de más de 60 años constituyen alrededor del $17 \%$ de la población chilena y representarán el 33\% en 2050, aumentando los mayores de 79 años de 2,6\% a $8,7 \%$ de la población. Desde la teoría de los ocho estadios del desarrollo psicosocial de E. Erikson, y su actualización y extensión hacia un noveno y último estadio denominado "gerotrascendencia", iniciado aproximadamente hacia los 75-80 años y hasta la muerte, se examinan los desafíos propios de la adultez mayor, se entregan algunas claves para una mejor calidad de vida en la vejez y algunas interrogantes para futuros estudios psicológicos en el tema.

\section{ABSTRACT}

Literature on old age (OA) in Chile is reviewed as part of normal psychological development, its characteristics and challenges,
Palabras clave: envejecimiento, política social, psicología, desarrollo humano. Keywords: ageing, social policy,

\footnotetext{
* Doctor en Psicología, Facultad de Psicología Universidad de Talca. ORCID: https:// orcid.org/0000-0003-2433-9271. WOS Researcher ID: G-9522-2012. Contacto: emoyano@ utalca.cl
} 
and socioculturally contextualized. A socio-demographic description of this growing group is presented and some central ideas about social policies in this regard are summarized. People over 60 years of age constitute about $17 \%$ of the Chilean population and will represent about $33 \%$ of the population in 2050 . People over the age of 79 years will increase from $2.6 \%$ to $8.7 \%$ of the population. The challenges of old age are examined based on E. Erikson's theory of the eight stages of psychosocial development, and its update and extension towards a ninth and last stage called "gerotranscendence", which begins approximately at 75-80 years of age and lasts until death. It provides some tips for a better quality of life in old age and asks some questions about future psychological studies on the subject.

\section{RESUMO}

Este artigo se trata de uma revisão de literatura sobre a terceira idade no Chile como parte do desenvolvimento psicológico normal, suas características e desafios, além de sua contextualização sociocultural. Uma caracterização sociodemográfica deste grupo crescente é elaborada e algumas ideias centrais das políticas sociais a este respeito são resumidas. As pessoas com mais de 60 anos constituem cerca de $17 \%$ da população chilena e representarão $33 \%$ em 2050, com um aumento de $2,6 \%$ para $8,7 \%$ da população com mais de 79 anos. Desde a teoria dos oito estágios de desenvolvimento psicossocial de E. Erikson, e sua atualização e extensão a um nono e último estágio chamado "gerotranscendência", que começa aproximadamente aos 75-80 anos de idade e dura até a morte, são examinados os desafios próprios da terceira idade, alguns pontos fundamentais para uma melhor qualidade de vida na velhice e algumas questões para futuros estudos psicológicos sobre o assunto. psychology, human development.

Palavras-chave: envelhecimento, política social, psicologia, desenvolvimento humano. 


\section{Introducción}

El envejecimiento de la población es un fenómeno social de alto impacto, que trae consigo desafíos para los gobiernos y la sociedad en su conjunto. Está determinado por tres componentes básicos de la dinámica poblacional: los nacimientos (fertilidad), las muertes (mortalidad) y las migraciones (Organización Mundial de la Salud, OMS, 2016). Así, la declinación de la fertilidad (menos nacimientos en relación a periodos anteriores) y una mayor esperanza de vida en las edades avanzadas son las dos causas principales de índole demográfica del envejecimiento poblacional. Hay 868 millones de personas mayores de 60 años en el mundo - cerca del $12 \%$ de la población total - y para 2050 está previsto que aumente a $21 \%$. La población de las Américas ha ganado 16 años de vida como promedio en los últimos 45 años (OPS, 2017). El incremento en la esperanza de vida será mayor y más rápido en los países en desarrollo como Chile, donde se prevé que la población de adultos mayores se cuadruplique en los próximos 50 años. Por cierto, la actual pandemia puede afectar esto.

La ciencia que estudia las enfermedades en la adultez mayor (AM) es la geriatría, que es una especialidad médica; mientras que la que estudia la salud, la psicología y la integración social y económica de las personas que se encuentran en la vejez es la gerontología, ejercida por diferentes especialistas no médicos y médicos. Así y consecuentemente, en la gerontología se practica un enfoque integral, considerando creencias y valores propios de la época respecto de la AM, y cómo ello influye en las construcciones que una sociedad produce para comprender el envejecimiento y la vejez (Yuni y Urbano, 2008).

El envejecimiento, más allá de su sustrato biológico, es un fenómeno sociocultural que adopta la forma que cada cultura le otorga. En este estudio se busca dar cuenta, de un modo general, de las características sociodemográficas del colectivo de adultos mayores (As Ms) chilenos, las políticas gubernamentales de los últimos gobiernos al respecto, las características psicológicas de la AM según una teoría consolidada y en expansión del desarrollo humano, y sugerencias para futuros estudios.

\section{Método}

Se trata de un estudio documental, realizado mediante la revisión de la Web of Science (WoS) de los últimos diez años (2010-2019), desde la 
que se analizó literatura internacional pero principalmente literatura local pertinente, para caracterizar sociodemográfica y globalmente la población de adultos mayores en Chile, los estereotipos que le afectan y las políticas que diferentes gobiernos han formulado para atenderla. Se recoge de una teoría consolidada del desarrollo humano (Erikson, 1970, 2000) las características centrales de las últimas etapas del desarrollo correspondientes a la adultez mayor (60 y más años), incluyendo una novena etapa recientemente agregada, para finalmente proponer futuros estudios para la psicología social y del desarrollo al respecto.

\section{Resultados}

Serán descritos en cinco acápites, referidos respectivamente a la cultura y estadísticas generales acerca del envejecimiento en Chile, sobrevuelo a políticas sociales para la AM, el desarrollo humano y las etapas de la AM, algo sobre pérdidas y longevidad, para concluir formulando sugerencias para futuros estudios.

\section{Cultura y estadística general acerca del envejecimiento}

Como fue adelantado, el envejecimiento, mucho más que un fenómeno biológico ${ }^{1}$, es en realidad y eminentemente un fenómeno cultural o social, que adquiere la forma, matices y representación social propios de la cultura en la cual el adulto mayor está situado.

Cada cultura tiene su propio "envejecimiento" y modo de representarse socialmente expectativas y roles acerca de los As Ms. En Occidente, en los siglos $\mathrm{V}$ al $\mathrm{XV}$, las guerras, pestes y la pobre condición de vida impedían que la vida se extendiera por más de 45-50 años. La representación respecto del adulto mayor (A M) fue positiva durante la edad media hasta su final y siglo XV, pero el Renacimiento trajo una juventud reivindicativa relegándolo como símbolo de decadencia y muerte, y siendo atacado como nunca antes. El siglo XVII trajo mejor imagen del adulto mayor, pero, desde fines del XVIII hasta mediados del XIX, grandes transformaciones en Europa hicieron que los As Ms

1 Biológicamente, es la pérdida progresiva de la capacidad de las células de auto regenerarse, pero no hay consenso acerca de cuándo comienza. Algunos sostienen que alrededor de los 40 años, otros que alrededor de los 26-30 años, e incluso algunos sostienen que desde el momento en que se nace. 
proletarios no pudieran mantener los esfuerzos físicos demandados por nuevas formas y ritmos de producción, siendo desplazados del trabajo, cayendo en la miseria, y muriendo (Martínez Ortega et al., 2002).

La representación social del A M en muchos países orientales es positiva. Éste es percibido como viviendo una etapa de máximo desarrollo, experiencia y sabiduría, considerándosele con absoluto respeto y preferencia. Contrariamente, en muchos países occidentales la representación es frecuentemente negativa, conceptos como "viejo"(a), o infantilizadores ("abuelito/a"), hasta denominaciones menos negativas, tales como "jubilado o retirado" para hombres, y de "pensionada" para mujeres, son muy frecuentes.

Muchas formas de denominación del A M encierran estereotipos, los que han sido identificados en diferentes culturas occidentales, incluida la latinoamericana, y siempre con un contenido negativo (Lasagni et al., 2013). En 1968, Butler formuló el concepto de "viejismo" o "edadismo" (ageism) para referirse al conjunto de estereotipos y discriminación contra las personas debido a su mayor edad (Salvarezza, 1988). Alrededor de esos años también, en un ensayo marxista acerca de la vejez, de Beauvoir (1970) afirmó que la imagen del A M en la sociedad occidental se debatía entre la del "viejo loco" y la del "viejo sabio", de modo que, ya sea por su virtud o por su abyección, lo sitúan fuera de la sociedad. Así, el paradigma característico de nuestra época es el denominado "viejismo", concibiéndose la vejez desde lo deficitario, desde la carencia, poniendo el acento en el declive físico y mental del A M, con una imagen de incapacidad e inutilidad social (Carmona, 2012). Esto es prejuicioso y muy perjudicial para el A M, ya que propicia su exclusión, discriminación y dependencia (Grijalva et al., 2007). Los estereotipos y estigmas asociados a los conceptos de vejez y envejecimiento afectan generalizadamente a los ciudadanos, incluyendo a los profesionales de la medicina y de la salud en general. Ejercen influencia consciente o inconsciente, directa o indirecta, no solo en nuestras actitudes y comportamientos al interactuar con As Ms, sino que también en la toma de decisiones en lo público y en la construcción de políticas sociales (Berriel et al., 2017).

Las estadísticas actuales acerca del A M en Chile muestran que en 1990, con poco más de 13,2 millones de habitantes, la esperanza de vida era de 73,7 años, y hoy, con alrededor de 18,7 millones (50,6\% 
mujeres), alcanza más de 80 años (OPS, 2017), ubicando a Chile en el lugar 36 en un ranking de 192 países (mujeres 82,09 años y hombres 77,18 años). Han contribuido a ello la mejora en las condiciones de vida, incluyendo progresos en la medicina, mejor cobertura de los servicios sanitarios, mayor autocuidado consecuente, disminución de las tasas de natalidad, fecundidad y mortalidad (Chackiel, 2004; Thumala et al., 2015).

En los últimos cincuenta años, la población de jóvenes chilenos menores de 15 años se redujo a la mitad y la de As Ms se duplicó, y los mayores de 60 años, que hoy corresponden al $17 \%$ de la población nacional, en 2050 representarán el 33\%, mientras que los mayores de 79 años subirán de 2,6\% a 8,7\% de la población (Arnold et al., 2018; Díaz, 2017). Según reportes de 2017, la cantidad de As Ms en Chile es 3.439.599 personas, de los que el $56,6 \%$ son mujeres $(43,4 \%$ hombres).

Desde la perspectiva de la medicina, típicamente circunscrita a la enfermedad, se estudia el nivel de dependencia funcional del A M, es decir, el grado en que necesita de otros para llevar a cabo sus actividades diarias o bien, el grado de dificultad que le genera realizarlas. También, la evaluación de la demencia (multiforme, siendo el Alzheimer frecuente) (Quiroga et al., 2004), cuyo estudio es de interés creciente, y afectando aproximadamente a 180.000 mil chilenos(as) (Slachevsky, 2015). Según la encuesta CASEN (2017), una mayoría de los As Ms en Chile $(85,8 \%)$ no presenta dependencia funcional, un $4,3 \%$ presenta dependencia severa, un $5,5 \%$ moderada y un $4,4 \%$ leve.

No existe un único modo de definir cuándo comienza el proceso de envejecimiento. Aquí adscribimos a la definición de la Asamblea Mundial sobre el Envejecimiento (Naciones Unidas) para la cual el A M es aquella persona cuya edad oscila entre 60-80 años (al mayor de 80 años lo denominó anciano) y envejecimiento es el conjunto de cambios fisiológicos que ocurren, en un organismo a través del tiempo (OMS, 1989), lo que incluye cambios de naturaleza bioquímica, morfológica, social, psicológica y funcional. Por otra parte, siguiendo el criterio de "edad social", se considera que una persona ha llegado a la AM cuando presenta ciertas opiniones, creencias y conductas, así como cuando ocupa lugares en la sociedad que en un determinado contexto social e histórico se atribuyen a este grupo de personas, por ejemplo, cuan- 
do se jubilan (Carmona, 2012). En algunos países e instituciones existe presión e incluso obligatoriedad a que el AM jubile, incluso si están todavía en condiciones para trabajar, lo cual contribuye a su precariedad económica, su marginación social y, así, a su vulnerabilidad. Esto representa discriminación y una transgresión del concepto legal que la jubilación es un derecho más no una obligación.

\section{Sobrevuelo a políticas sociales para la adultez mayor}

Las políticas sociales son un conjunto de decisiones, acciones e intervenciones desarrolladas por el Estado, relacionadas con un objetivo público definido, y que tienen como propósito mejorar la calidad de vida (CV) de la población, promover la integración social, fortalecer la gobernabilidad del país y reducir la pobreza y desigualdades sociales (Astete y Vaccari, 2017; Lahera, 2008; Ministerio de Planificación y Cooperación, MIDEPLAN, 2000).

Como es sabido, las políticas sociales se llevan a cabo por medio de programas sociales, los que, a su vez, se operacionalizan en proyectos. Los programas sociales son un "conjunto integrado y articulado de acciones, prestaciones y beneficios destinados a lograr un propósito específico en una población objetivo, de modo de resolver un problema o atender una necesidad que la afecte" (Ley ${ }^{\circ}$ 20.530, Biblioteca del Congreso Nacional de Chile, 2011, pp. 1-2). Es el Estado el agente que, por medio de políticas sociales, debe velar por el bienestar y CV de las personas, protegiendo a los sectores más desfavorecidos (Ganga et al., 2016).

Cuatro principales roles ha cumplido el Estado chileno respecto de los As Ms. durante el s. XX. Primero, el Estado protector desde finales del s. XIX hasta 1930, centrado en la clase trabajadora y proletaria, concibió los problemas sociales como un tema de justicia. Segundo, un Estado benefactor - 1930 a 1955 aproximadamente-, benefició principalmente a sectores medios, gracias también a una ampliación de los beneficios sociales. Tercero, el Estado redistribuidor -1964 a 1973-, que procuró la redistribución de los ingresos en beneficio de los sectores más desfavorecidos. Cuarto, el Estado subsidiario -desde la dictadura 1973 a 1990, pasando por la recuperación de la democracia, y gobiernos habidos en el siglo XXI hasta ahora(Dávila León 1998). 
Como es sabido, durante la dictadura cívico-militar (1973-1990) fueron privatizadas y mercantilizadas ciertas áreas de la política social, y con los gobiernos de la Concertación - 1990 a 2010 - se mantuvo la lógica de la responsabilidad individual de las personas para suplir sus propias necesidades, aunque se incrementaron los recursos o el gasto social. Se clasificó a las personas en tres categorías: las que pueden por sí mismos satisfacer sus carencias; las que poseen la capacidad pero no les es suficiente, por lo que requieren de la ayuda estatal y, finalmente, los que necesitan de la asistencia del Estado para sobrevivir, los cuales corresponden a las personas en situación de pobreza o pertenecientes a un grupo vulnerable. Desde esta lógica, las políticas sociales se orientan principalmente a las personas del tercer grupo (Arteaga e Iñigo, 2015), focalizándose en ellos la entrega de bienes, beneficios y, a su vez, obteniéndose un grado de control social (Alfaro, 2012).

Para algunos, estas políticas y programas sociales se fundan en una visión asistencialista, cuyo objetivo es la disminución de los principales problemas de los grupos vulnerables y la satisfacción de sus carencias básicas. El enfoque sería individualista, centrado en las personas o en sus familias, desconsiderando eventuales intervenciones enfocadas hacia la comunidad o colectivos de la población en general (Alfaro y Zambrano, 2009; Reyes et al., 2015). Así, las políticas sociales hasta aquí no habrían promovido la participación ni la gobernanza (Alfaro y Martín, 2015), concibiéndose a las personas como sujetos dependientes, receptores de los beneficios otorgados por el gobierno, sin el derecho de exigir o demandar (Freudenberg \& Tsui, 2014). Consecuentemente, los planes y programas desarrollados desde estas políticas son formulados sin la participación de los ciudadanos y sin el conocimiento territorial de la comunidad, sino más bien desde la tecnocracia del Estado (Alfaro y Martín, 2015), y de modo altamente centralizado desde la capital del país hacia el resto del territorio nacional.

En los últimos años se incluye en las políticas la idea de que las personas y sus comunidades pueden y deben intervenir activamente en la elaboración y posterior ejecución de los planes y programas. Sin embargo, ello casi no se materializa en la práctica y, en salud, por ejemplo, las culturas institucionales médico-organizacionales facilitan escasamente la aplicación de enfoques más comunitarios en el 
abordaje preventivo y curativo en salud. Los programas que integran la participación de los ciudadanos representan una minoría respecto del total vigentes en la actualidad (Reyes, Olivares, Berroeta y Winkler, 2015). Las políticas sociales enfocadas hacia la vejez, en el contexto neoliberal, tienen fundamentos similares a los de las políticas sociales en general, con esfuerzos programáticos de carácter asistencialista y focalizados en la satisfacción de necesidades básicas de individuos y no en la comunidad (Reyes et al., 2015), desconsiderando hallazgos que afirman la importancia de ésta para alcanzar un envejecimiento exitoso (Varela, 2016).

En una mirada sintética de la evolución de las políticas para los As Ms de las últimas tres décadas, se puede resumir que, con la recuperación de la democracia, durante el gobierno de Patricio Aylwin (19901994) se formuló un primer documento, identificando la necesidad de abordar la vejez: "Política Nacional sobre Envejecimiento y Vejez" (MIDEPLAN, 1993). Según Caro (2014), el mérito principal de dicha política consistió en introducir en la agenda pública el tema del A M, aunque, en la práctica, desde tal documento no fueron desarrollados programas sociales. El gobierno siguiente - de Eduardo Frei Ruiz-Tagle (1994-2000) - constituyó la Comisión del Adulto Mayor y se declaró al Estado como el principal promotor del cambio, y con un rol subsidiario (Caro, 2014). Esta comisión dio paso en 1996 a la creación del Comité Nacional para el Adulto Mayor que, a su vez, dio origen a la Ley $\mathrm{N}^{\circ} 19.828$, mediante la cual fue creado el Servicio Nacional del Adulto Mayor (SENAMA) en 2002. La creación y posteriores acciones de este servicio ilustran el proceso de consolidación de la institucionalidad como el eje central de las acciones estatales para la vejez en la última década (Huenchuan et al., 2007).

El gobierno de Ricardo Lagos Escobar (2000-2006) actualizó la Política Nacional para el Adulto Mayor (SENAMA, 2004), buscándose infundir valores como equidad, solidaridad y ejercicio de derechos, así como incluir a la comunidad y la familia en la búsqueda del bienestar de las personas mayores. Michelle Bachelet, en su primer gobierno (2006-2010), dio un giro y procuró afirmar un enfoque de derechos en las acciones estatales (Huenchuan et al., 2007), creándose los Consejos Asesores Regionales de As Ms (2008), incluyendo a representantes de sus organizaciones de cada región, buscándose el ejercicio activo de 
ciudadanía, así como la protección de los derechos de los As Ms (Roqué et al., 2016).

En el primer gobierno de Sebastián Piñera (2010-2014) fue anunciada la puesta en marcha de la Política Integral de Envejecimiento Positivo, resultante de un trabajo de amplia participación, incluyendo un equipo multidisciplinario de profesionales del ámbito público y privado, un Comité Interministerial, diferentes Servicios y Subsecretarías nacionales, As Ms de organizaciones de la sociedad civil, informes técnicos nacionales e internacionales, así como políticas para el A M vigentes en otros países (SENAMA, 2012).

Durante el segundo gobierno de Michelle Bachelet (2014-2018) fue reafirmado el enfoque de derechos, y ratificada la Convención Interamericana sobre la Protección de los Derechos Humanos de la Personas Mayores. Se promovió el respeto y ejercicio de derechos de los As Ms, así como también se visibilizó la responsabilidad de los Estados en su bienestar (SENAMA, 2017a).

El Programa de Gobierno de Sebastián Piñera Echeñique (20182022) mantiene adscripción al concepto de "envejecimiento positivo" y busca reconocer y visibilizar a los As Ms y sus derechos, potenciando su identidad cultural y social, fomentando la participación activa en la sociedad, aumentando los factores protectores de su salud, retrasando la institucionalización de los dependientes, promoviendo la corresponsabilidad en el cuidado de la salud, generar alianzas público-privadas y reconocer un enfoque socio-sanitario para enfrentar la situación. También compromete un mejoramiento sustantivo de las pensiones.

Toma la propuesta de SENAMA 2012, buscando proteger la salud funcional de las personas mayores, mejorar su integración en las diversas esferas de la sociedad y aumentar los niveles de bienestar subjetivo de la población mayor. La incorporación o reforzamiento de la búsqueda del bienestar y de mejorar la CV de los ciudadanos objeto de las políticas, son objetivos centrales planteados desde la psicología y ciencias sociales desde hace tiempo. Y, aparentemente, el bienestar de los As Ms está muy asociado a la disponibilidad y acceso a instituciones de salud (Sánchez et al., 2017).

Uno de los programas dirigido a As Ms autovalentes de 60 años o más es el de "envejecimiento activo", desarrollado por SENAMA desde 
2013. Se busca promover participación en actividades para envejecer activamente, a través del equilibrio entre la responsabilidad personal, el encuentro y la asociación, la solidaridad entre generaciones y el diseño de entornos que favorezcan la CV y retrasen la aparición de dependencia (Roqué et al., 2016). Se ofrecen talleres, jornadas y seminarios para fortalecer habilidades, y eventos masivos de difusión del buen envejecer. Los ámbitos de acción del programa se relacionan con la actividad física y recreativa, así como con la cultura y las artes (SENAMA, 2017b). Fernández et al. (2018) probaron un modelo explicativo del envejecimiento activo, mostrando que la funcionalidad física y cognitiva, y variables asociadas a la tonalidad afectiva aparecen como causas del mismo, mientras que la ausencia de sintomatología depresiva y la condición de activo en el mercado laboral son indicadores de este envejecimiento, en los que las variables sociales mostraron la mayor capacidad predictiva.

El concepto de CV está referido con altísima frecuencia en las políticas y programas. CV en salud, educación y seguridad especialmente, que son los servicios que debiera proveer el Estado, cuando no es un Estado precario o fallido. En psicología se operacionaliza la CV como bienestar (Moyano et al., 2010) mientras que, para Ardila, CV es:

Un estado de satisfacción general, derivado de la realización de las potencialidades de la persona... Es una sensación subjetiva de bienestar físico, psicológico y social. Incluye como aspectos subjetivos la intimidad, la expresión emocional, la seguridad percibida, la productividad personal y la salud objetiva... Entre los elementos objetivos se encuentra el bienestar material, salud objetiva y las relaciones armónicas con la comunidad y con el ambiente, mientras que entre los subjetivos considera el sentirse sano, productivo, seguro, expresar emociones y la intimidad como aspectos que conllevan a la CV de una persona. (2003, p. 163)

Un síntesis de la condición socioeconómica del A M en Chile permitirá al lector formarse su propia opinión respecto de algunos aspectos de su CV. La Encuesta de Caracterización Socioeconómica del año 2017 (CASEN, 2017) y algunas otras referencias servirán para ello.

En 2017 los As Ms conforman una población de 50,7\% de casados, $20,9 \%$ viudos, $7,3 \%$ separados/divorciados, $12 \%$ solteros, $8,8 \%$ 
conviviente con o sin acuerdo civil y $0,3 \%$ anulados. Su distribución porcentual según ingresos es bastante homogénea, distribuyéndose similarmente entre los quintiles de menor (I) a más alto ingreso (V); así, alrededor del $22 \%$ se ubica en los quintiles I y II, aproximadamente un $21 \%$ en el III, diferenciándose el porcentaje correspondiente a los ingresos más altos con un 15,4\% (CASEN, 2017). La responsabilidad de jefatura de hogar que recae sobre los As Ms asciende desde 24,9\% en 1990 a $36,3 \%$ en 2017 , a nivel nacional, con un 53,9\% para hombres y $46,1 \%$ para mujeres en lo urbano, y del $66,7 \%$ urbano versus $33,3 \%$ para lo rural, respectivamente. Según tipología familiar, la nuclear biparental es dominante con un $53,4 \%$, mientras la intergeneracional comparada para 1990 y 2017, muestra que las familias de tipo multigeneracional (tres o más generaciones reunidas) disminuyeron del 7,3 al 5,3\%, aumentándose del 4,8\% al 11,7\% el tipo correspondiente a la generacional conformadas solo por personas As Ms sobre 64 años. Esto último aumenta la probabilidad de soledad o aislamiento del A M.

La incidencia de la pobreza según ingresos en los As Ms en Chile se habría más que duplicado si no existiera el Pilar Solidario, a cargo exclusivo del Estado. Éste ha desembolsado US\$ 11 mil 600 millones para financiar el Sistema de Pensiones Solidario (SPS) que ayuda a los As Ms más pobres del país, un universo de poco más de dos millones de personas. La carencia por ingresos en la población de 65 años llegaría a $14,1 \%$ sin los subsidios, mientras que la vulnerabilidad en ese grupo habría aumentado desde el 19,2 a un 28,3\%. A 2017, con un sueldo mínimo legal de 264 mil pesos, el 57\% de los As Ms tiene una jubilación promedio de $\$ 130.927$, mientras un $43 \%$ promedia $\$ 298,616$ (Fundación SOL, 2017). Así, si los As Ms carenciados contaran solo con el ingreso de sus pensiones, estarían en una condición de pobreza extrema. La participación del A M en la pobreza total disminuye proporcionalmente más que en otros tramos de edad, contribuyendo solo con $1 \%$ en la incidencia de la pobreza extrema. Los As Ms no tienen ningún impacto negativo e incluso ayudan a disminuir la pobreza con sus aportes, aparte de su relevante contribución de apoyo y red social para la familia en general, en la que particularmente las mujeres destacan históricamente por su labor de cuidado a terceros.

Del conjunto de indicadores de la denominada "pobreza multidimensional”, el más deficitario en los As Ms corresponde a educación 
- escolaridad-, siendo quienes más analfabetos concentran, duplicando al grupo inmediatamente anterior (45-59 años), y triplicando su porcentaje en territorio rural respecto del urbano. Sin perjuicio de esto, en su conjunto la población analfabeta de 60 años y más ha disminuido de 15,2 a $8,2 \%$. Aunque la escolaridad de los As Ms es la más baja entre todos los tramos de edad (diferencias estadísticamente significativas), sus años promedio de escolaridad aumentaron de 6 en 1990 a 8,3 años en 2017 (CASEN, 2017). En las regiones de Valparaíso, Ñuble y Maule la proporción de As Ms es ligeramente mayor que el promedio nacional en Chile; con 23,3\%, 22,6\% y 21,9\% respectivamente.

Es sabido que los hombres mueren antes que las mujeres. Así, la población de As Ms es mayoritariamente de mujeres, quienes reportan peor salud general y mental, y más sentimientos negativos que los hombres (Moyano-Díaz \& Mendoza-Llanos, 2021), así como también menos satisfacción de vida (Alvarado y Plaza, 2014). Las mujeres chilenas participan menos de la fuerza de trabajo normal, ocupan menos cargos de responsabilidad y obtienen menos ingresos, debiendo enfrentar desventajas de menor educación o mayor analfabetismo, junto con mayores índices de viudez y soledad, todo lo cual afecta su eventual jubilación y su AM (CASEN, 2017). En otras latitudes - y aparentemente también en Chile-, los hombres llevan su vida teniendo una menor probabilidad de recibir ayuda económica de parte de sus hijos, una mayor probabilidad de endeudarse y otros problemas financieros, y, en general, una más baja satisfacción con su situación financiera (Sobieszczyk et al., 2003). Ha sido sugerido que las diferencias vinculadas al género entre los As Ms, estarían relacionadas con experiencias tempranas de vida, roles y estructuras de recompensa, particularmente acceso a jubilaciones y otros tipos de apoyo. Las inequidades de género durante la vida - v.g., remuneraciones más bajas para mujeres, periodos que, por causa de embarazo, crianza de hijos o cuidado de terceros, han interrumpido su trabajo remunerado- les lleva a jubilaciones más precarias y, así, a una vejez más vulnerable que sus contrapartes masculinas, todo lo cual podría explicar diferencias en las evaluaciones de su CV (CASEN, 2017).

En Chile, un $63,8 \%$ de las mujeres y un $71,1 \%$ de los hombres As Ms de 65 y más años informan que su CV es buena o muy buena, y un $4,5 \%$ de aquellas contra solo un $2,0 \%$ de los hombres que es mala o 
muy mala. También, un 56,8\% de mujeres y un 39,6\% de hombres declaran que su salud es regular o mala. Cuando reportan su satisfacción con la vida, las mujeres chilenas están satisfechas, con un promedio de 6,9 puntos sobre 10 y los hombres 7,2 (diferencia estadísticamente significativa). Éstos han estado globalmente más satisfechos durante la mayor parte de su vida hasta los 79 años, edad a partir de la cual las mujeres les alcanzan y les superan desde 90 años y más. En Magallanes y Aysén los As Ms alcanzan el mayor nivel de satisfacción (7.4/10), mientras en Maule el menor (6.4/10) (Alvarado y Plaza, 2014). Similarmente a Chile (Moyano y Ramos, 2007; Alvarado et al., 2016), la evidencia de otros países muestra que quienes conviven - aun sin mediar casamiento formal- o son casados, experimentan más bienestar que quienes no (Sobieszczyk, et al., 2003), acentuando así la importancia de la compañía y apoyo social para la estabilidad psíquica, el bienestar personal y el desarrollo humano.

El desarrollo emocional durante la vida sigue una trayectoria diferente a la del biológico y cognitivo, ya que mientras éstos decrecen o se fragilizan con la edad, el A M experimenta ganancias en control emocional y un incremento en la importancia relativa de la información emocional en el procesamiento de la información (Márquez-González et al., 2004).

\section{El desarrollo humano y la etapa de la adultez mayor}

El desarrollo humano es un proceso muy complejo, que resulta de una dialéctica entre biología y cultura. Incluye diferentes ámbitos o subsistemas en interacciones entre sí y con el ambiente físico y social en que cada organismo crece y evoluciona. El desarrollo psíquico es un proceso de integración armónica de diferentes subsistemas en crecimiento que, influido por las leyes de la maduración (adecuada conformación física global y cerebral en particular), la diferenciación (de partes y funciones corporales y psicológicas), y la especialización (habilidades físicas y mentales) y su perfeccionamiento, se cierra con la muerte (Oerter, 1980). Así, en psicología, desde antaño se concibe el desarrollo no como mero "crecimiento" -físico o cerebral-, sino como una integración armónica de desarrollos físicos y mentales diferentes: psicomotor, emocional o afectivo, sexual, del pensamiento o cognitivo y social. Globalmente dicho, se transita desde un polo bá- 
sico del descontrol de impulsos y deseos, pasando por el aprendizaje y desarrollo del control, hasta - y eventualmente- la ataraxia, como en Jesús, Buda y otros grandes iniciados.

Entre las teorías del desarrollo humano, la del desarrollo psicosocial de E. Erikson (1968,1974, y revisión en 1996 por Joan Erikson, 2000) estableció inicialmente que el ser humano evolucionaba a lo largo de ocho etapas, que revisiones actuales extendieron a nueve, consistente con la mayor expectativa de vida hoy. Para Erikson, el ser humano progresa en función de estímulos biológicos provenientes de su organismo, en inter juego con la sociedad-cultura o ambiente donde nace, crece y se humaniza.

Las nueve etapas de la teoría eriksoniana tienen diferentes duraciones, cierta variabilidad entre personas y, por lo tanto, al señalar edades para cada etapa se trata de referencias aproximadas, nunca exactas. Las primeras cinco etapas del desarrollo psicosocial humano (tres infancias, más pubertad y la adolescencia) son más breves que las siguientes cuatro etapas, propias de la adultez: adultez joven, adultez madura, adultez mayor y gerotrascendencia. Las nueve etapas llevan como nombre el tema central o crisis o desafío a resolver, y sus edades aproximadas son (Erikson, 1974): 1) Confianza versus Desconfianza (nacimiento a 18 meses); 2) Autonomía versus Vergüenza y duda (19 meses a 3 años); 3 ) Iniciativa vs. Culpa (3 a 5 años), 4) Laboriosidad vs. Inferioridad (6-7 a 12 años), 5) Identidad vs. Confusión de roles (adolescencia hasta los 20-25 años), 6) Intimidad vs. Aislamiento (20-25 a 40 años). 7) Generatividad vs. Estancamiento (40 a 60 años), también denominada adultez intermedia; 8) Integridad del Yo vs. Desesperación (60-65 a 75 años) y 9) Gerotrascendencia (75-80 años y más, hasta la muerte) (Brown y Lowis, 2003).

Un concepto central en la teoría es la identidad, entendida como el conjunto de rasgos o características de una persona que permiten distinguirla de otras. La identidad también es la concepción que tiene una persona -o un colectivo, v.g. los As Ms- sobre sí mismo en relación con otros. La identidad individual experimenta cambios a lo largo de la vida y, eventualmente, quiebres o rupturas abruptas o traumáticas, al vivir situaciones extremas que pueden afectar su desarrollo. Así, la pobreza - como principal condición estresante-, la orfandad, las guerras, dictaduras, persecución, encarcelamiento, tortura y situaciones extre- 
mas tienen una enorme capacidad de alterar identidades inicialmente “intactas". Amenazas actuales a la identidad de los niños y jóvenes son frecuentes por medio de las "redes sociales" — de amplia y veloz cobertura - para acosar o difamar a sus pares u otros, lo cual puede conducir a sus víctimas a alteraciones de su identidad, tristeza, depresión e incluso — cada vez más penosa y frecuentemente - al suicidio. La pusilanimidad social, política e institucional-legal, normativas penales atrasadas, el individualismo del "sálvese quien pueda" y otras características de sociedades laxas o francamente anómicas, contribuyen a "naturalizar" este tipo de violencia atentatoria a la estabilidad y salud mental.

La identidad se desarrolla a lo largo de las diferentes etapas del ciclo vital y cada una se caracteriza por una "crisis psicosocial" (o tensión) que, en términos positivos, podemos designar como "desafíos", los cuales deben ser superados para acceder a la siguiente etapa. Por ejemplo, un punto de inflexión de la identidad social o desafío vital significativo que el AM debe afrontar, y que está normativamente pauteado, es la jubilación. El retiro del trabajo significa un cambio de identidad personal y social de transición, o pasaje de ser "activo" a "pasivo", de "trabajador" a "pensionado". Además de la precariedad del ingreso que trae consigo este nuevo estatus en Chile, el cambio exige una reestructuración de un nuevo rol y, así, un cambio identitario personal y social de alta repercusión y significado.

El desarrollo humano en cada etapa depende de la maduración (biológica) o cambios en este nivel, pero muy especialmente de la interacción social con otros individuos y con el ambiente o entorno mayor. La necesidad de relación con los otros debiese contribuir a prácticas sociales de intercambio que promuevan la construcción de una sociedad con relaciones de confianza, igualitaria, amable y fraterna que, entre otras cualidades, son las que tienen menos criminalidad y mortalidad, y las mejores para vivir la AM (países del norte de Europa, v.g. Noruega o Dinamarca). Esto por cierto no deviene "naturalmente", se requiere un esfuerzo social, educativo y político mayor, expresamente dirigido (del cual estamos muy lejos, sin observarse iniciativas al respecto). Un atributo de la interacción social que los pueblos latinoamericanos mayoritariamente conservan es la afabilidad, simpatía o relación amistosa de familiaridad, y gusto por las "convivencias" en los lugares de trabajo o en las familias, en barrios o vecindarios. Las per- 
sonas se detienen en su cotidianeidad para conversar con otro(s) por el simple hecho de disfrutar haciéndolo, preguntando e intercambiando acerca de amigos o conocidos comunes, familia, política, noticias u otros. Este estilo afiliativo es fuente importante de bienestar (MoyanoDíaz, Mendoza-Llanos \& Páez, 2021) y probablemente contribuya a explicar por qué los latinoamericanos, no obstante disponer de menores recursos económicos, puntúan semejante en satisfacción vital y felicidad que ciudadanos de países con economías más ricas.

Durante la etapa de la adultez (con inicio alrededor de los 20 o 25 años, dependiendo de si hay autonomía por la incorporación al trabajo o no), el individuo alcanza su mayor productividad y rendimiento. En la especie humana - y en su actual estado de desarrollo cultural- se considera "adulto" a quien, además de tener capacidad de reproducción y apogeo de sus capacidades físicas, ha alcanzado discernimiento (capacidad de distinguir entre el bien y el mal), ha madurado afirmando su personalidad y ha alcanzado estabilidad psicológica. Para la teoría eriksoniana la adultez es el periodo más extenso del desarrollo humano, subdividiéndose en cuatro etapas (6, 7, 8 y 9), la primera de las cuales comienza inmediatamente después de la adolescencia (25 años y más), y la última alrededor de los 75-80 años, terminando con la muerte (Erikson, 2000). La etapas 8 y 9 conforman la denominada adultez mayor (AM), tema principal aquí.

Para Erikson, el sentido de identidad proporciona la capacidad de experimentarse a sí mismo como alguien que tiene continuidad y semejanza, y para actuar en consecuencia. El término "crisis psicosocial" puede ser entendido como desafío, tema, o "cuestión central" propio de cada etapa, que cada quien deberá resolver. Dependiendo de cuán adecuadamente lo haga, transitará más "exitosamente" a la etapa siguiente. Aunque la crisis tiene un sustrato fisiológico, ella es fundamentalmente una solicitud o demanda social externa en función de la edad, aquello que la sociedad impone al ser humano en sus diferentes edades. Así, de la interacción entre maduración (biológica) y procesos sociales de demanda surge el desafío-crisis o tema a resolver en cada etapa. Estas crisis-desafíos centrales tienen manifestaciones conductuales, pero esencialmente consisten en fenómenos encubiertos (o íntimos) de afrontamiento y de reflexión, en los que el ser humano desarrolla su identidad, madura, evoluciona, se perfecciona, supera sus 
defectos, consiguiendo tornarse un ciudadano aportador de bien a los demás, y a la construcción de una sociedad mejor (Erikson, 1968, 1974, 2000). Aquí estamos interesados en las etapas 8 y 9 , correspondientes a lo que se denomina AM, e introduciremos a ellas a continuación.

\subsection{Integridad del Yo versus Desesperación (octava etapa, adultez} mayor aprox. 60-75 años mujeres, 65-75 años hombres)

También llamada "edad de la sabiduría" (actuación con sensatez, prudencia o acierto). En la cosmogonía africana se distingue tres etapas de desarrollo humano: la del aprendizaje (infancia y juventud), la de la producción (adultos) y, la tercera y última, la de la sabiduría (vejez) etapa

...a la que todos aspiran (...) los ancianos ocupan una posición privilegiada porque constituyen el vínculo entre los vivos y los antepasados muertos. Para el hombre común la tercera edad es invierno, para el hombre sabio en cambio es la estación generosa de la cosecha, del saber dar y de la tranquilidad Espiritual, porque se vive en paz con lo que es imposible cambiar (Acerbi, 2013, p. 72)

Según la teoría de Erikson (2000), en esta etapa el A M revisa su historia, recorre su biografía, hace balances, tiene la posibilidad de desarrollar proyectos que siempre quiso y que las circunstancias lo impidieron; puede reconciliarse con su pasado, perdonar a quienes le han causado disturbio o mal, y también puede perdonarse. Puede lamentar no haber hecho suficiente, desesperarse porque no consiguió construir una familia con progenie adecuadamente socializada (buenos ciudadanos), o no consiguió él mismo ser un ciudadano que hizo algo que lo haga sentirse satisfecho, ya sea por su trabajo o por la comunidad. Si su evaluación de lo conseguido hasta allí es negativa, caerá en desesperación, experimentará ansiedad, angustia, tendrá serias dificultades para redefinir su rol y, así, experimentará disgregación, dispersión, no integración de partes de su biografía, pesar. Si una persona siente que su vida ha sido un fracaso y que es demasiado tarde para empezar en una nueva dirección, se llenará de desesperación (Erikson, 1968). Esto le impedirá otorgar sentido a su existencia, significado a lo realizado, satisfacción por lo logrado. Se lamenta por no haber vivido como debería y experimenta el temor por el término de una vida irrealizada, en medio de una vejez infeliz, amarga, porque ya no hay más tiempo. Así, 
ante la imposibilidad de prolongar la vida, con la ilusión de vivirla de nuevo pero diferentemente, teme a la muerte y no consigue prepararse adecuadamente para despedirse.

La resolución exitosa de la octava etapa tiene lugar cuando el individuo puede aceptar lo que ha pasado antes como inevitable y satisfactorio y, también, puede aceptar sin temor que la muerte ocurra probablemente en un futuro no muy lejano. Esto resultará en ego-integridad. La persona que resuelve adecuadamente la crisis de esta etapa afronta la muerte sin miedo, manifestando la virtud que Erikson denomina "sabiduría" (Erikson, 2000). El A M, a diferencia de los más jóvenes, tiene más confianza en su control emocional y, muy probablemente, el logro de una mayor integridad del Yo le posibilita más espontaneidad y menos condicionamiento a convenciones sociales, tales como buscar mostrar una imagen ideal de sí mismo. Tiene un mayor interés en cuidar los vínculos interpersonales y, en su selección, busca las relaciones proveedoras de bienestar; logra mayor control, regulación y madurez emocional, prefiriendo niveles de activación emocionales moderados más que intensos (Arias, 2013). El A M suprime más que los jóvenes su expresión emocional, y algunos estudios sugieren que lo hacen especialmente con las emociones positivas (Márquez-González, Fernández de Trocóniz, Montorio Cerrato y Losada Baltar, 2008).

Es en esta etapa en la que el A M alcanza la edad con la que la mayoría de las sociedades occidentales legalmente le habilitan jubilar. Interrumpir la vida laboral llevada hasta allí es un principal hecho vital, y hoy en Chile ello es postergado debido a la precariedad de las pensiones. En nuestro país, el A M típico de mediados a fines del siglo XX esperaba con expectación positiva su cumpleaños 65 , si era de sexo masculino (o 60 años si era mujer), y entre sus expectativas $-\mathrm{y}$ luchas sindicales- estaba revindicar la reajustabilidad de su pensión al $100 \%$ de lo obtenido como remuneración como trabajador (la "perseguidora"). Hoy, en cambio, en que desde un sistema público de reparto se ha pasado a otro privado y de capitalización individual impuesto en dictadura, en 1981 (asociaciones de fondos previsionales, AFP), los trabajadores o empleados mayoritariamente temen la jubilación por la dramática caída del ingreso que implica (Stuardo, Zavala y Merino, 2016). La promesa de 1980-1981, de "jubilar cuando usted quiera, con el 100\% o al menos el $75 \%$ de su sueldo", se ha hecho trizas, constituyendo un 
engaño más a las generaciones correspondientes. Se ha reportado que esta esperanza hecha añicos se ha transformado en una experiencia emocional negativa, de temor, incertidumbre y tristeza (Fernández, González, Rosell y Campos, 2016).

\subsection{Gerotrascendencia (novena etapa, 75 y más años, hasta la muerte)}

Las etapas de la AM y de la gerotrascendencia pueden constituirse en las de la creación de las mejores obras de una persona. Acerbi (2013,p. 72), informa que Verdi compuso sus mejores óperas - v.g. Otelo- después de los 70 años, y tenía más de 80 cuando escribió Falstaff. Que Miguel Ángel inició el Juicio Final (Capilla Sixtina) a los 65 años, esculpiendo el Moisés más tarde y La Piedad alrededor de los 80 . Goethe, terminó el Fausto cuando tenía más de 80 años. Tiziano pintó hasta sus 98 años. Que muchos grandes artistas mostraron una vejez fecunda y creadora, como Degas, Rembrandt, Durero, Tintoretto, Goya, Monet, Leonardo da Vinci, Picasso, Cervantes, Beethoven, Tolstoi y otros. Víctor Hugo (1802-1885) decía: "En los ojos de los jóvenes vemos llamas, pero es en los ojos de los mayores donde vemos la luz".

Los egipcios consideraban la inmortalidad prerrogativa de los dioses, y los faraones no podían lograrla dada su condición de mortales, por lo cual debían practicar anualmente la ceremonia del rejuvenecimiento o fiesta del jubileo (Heb Sed), procurando alcanzar la edad de la sabiduría, es decir los 110 años. Se consideraba a los As Ms como "mágicos", y se les respetaba por la sabiduría que atesoraban, obtenida en el camino de una larga vida. "Eran preservados de los trabajos duros por sus amos o familiares, procurando que envejecieran con dignidad, rodeados del cariño de parientes y amigos" (Acerbi, 2013, p. 70). Si se ha conseguido superar positivamente la crisis central de la octava etapa, se alcanza la novena etapa denominada "gerotrascendencia", término provisto por el sociólogo sueco Tornstam (2005), quien la fundamentó mediante estudios en As Ms europeos.

La formulación de la gerotrascendencia sugiere que vivir la vejez y hacer frente a sus desafíos produce un cambio en la meta perspectiva, desde una visión materialista y racional a una más cósmica y trascendente. Esto sería normalmente acompañado de un aumento en la satisfacción con la vida. Tornstam (2005) sostuvo que la sabiduría 
está identificada con la gerotrascendencia, y difirió de la descripción de Erikson relativa a la fuerza de la sabiduría en la octava etapa. Para aquel los efectos del aumento de la trascendencia en la personalidad a menudo se malinterpretan como una separación negativa, pero cuando el paradigma de observación cambia, entonces el cuadro también cambia. La teoría psicosocial sostiene que el individuo que resuelve favorablemente la octava etapa de crisis y alcanza la egointegridad, mira hacia atrás con satisfacción sobre su vida pasada y ahora puede aceptar inevitablemente la muerte sin miedo (Erikson, 1980). Tornstam, sin embargo, creyó que el individuo, en el camino hacia la gerotrascendencia, mira hacia adelante y hacia afuera, más allá de sí o de lo propio. Esto puede implicar una disminución del egoísmo y un aumento en la cantidad de tiempo dedicado a la reflexión tranquila.

Habría un movimiento más allá de todo miedo a la muerte, en lugar de la mera aceptación de su inevitabilidad, y puede tener lugar una redefinición del tiempo, del espacio y de los objetos. Esto, además, sería libremente elegido y puede ser positivo, en contraste con la retirada negativa sugerida, por ejemplo, por Cumming y Newell (1961) en su debatida formulación de la separación o la desvinculación que haría el A M respecto de su entorno. Si adherimos a esta formulación de Tornstam, no habría que presionar al A M - y especialmente al gerotrascendente - a tener actividad física o social, ni a participar "activamente" de ciertas actividades sociales que se le propongan, sino más bien entender y aceptar su opción de quietud, contemplación y reflexión.

En la novena etapa de desarrollo los puntos de crisis previamente resueltos son nuevamente confrontados. Como en la ya muy lejana primera etapa del desarrollo humano, la confianza básica frente a la desconfianza básica por ejemplo, el elemento (ego) sintónico ${ }^{2}$-es decir, la confianza - apoya primero el potencial de desarrollo, desarrollando la fuerza de confianza del Yo cuando esta etapa se resuelve favorablemente. En la vida posterior, con una creciente conciencia de

2 La ego-sintonía es vivir experimentar o sentir que lo que a uno le ocurre está en sintonía o resulta coherente con lo que uno es (su identidad). La ego-distonía, al contrario, es sentir que algo que a uno le acontece está en desacuerdo con la propia identidad, resultando así incómodo, molesto, perturbador. 
deterioro mental y físico, el A M, cada vez más frágil, puede comenzar a perder la confianza en su capacidad para mantener la independencia. Así, nuevamente confronta una crisis implicando confianza-desconfianza. Joan Erikson relevó el "elemento (ego) distónico (es decir, desconfianza) en primer lugar, para destacar su prominencia y potencia" (Erikson, 1998 p. 196), y subrayó la importancia de reconocer ese conflicto y tensión, ya que podría ser una fuente de crecimiento y fuerza.

Respecto a la octava etapa de crisis - integridad del ego versus desesperación-, Joan Erikson sostuvo que las dificultades a las que se enfrentan las personas en sus 80 y 90 años implican una batalla constante con el elemento ego-distónico, y que la desesperación es enfrentada cotidiana o diariamente. Así, conseguir afrontar la desesperación y superarla cada día es bastante difícil, porque “...uno ya no puede tener el lujo de la desesperación retrospectiva” (Erikson, 1998 p. 113). Añadió que si los ancianos podían llegar a una conciliación con los elementos inconfortables o molestos en sus experiencias de vida en la novena etapa, podrían avanzar con éxito en el camino hacia la gerotrascendencia.

Desde el punto de vista de su funcionamiento cognitivo, los As Ms parecen proclives a utilizar una "pragmática cognitiva" (Baltes \& Baltes, 1990) para permitir a la personalidad seguir creciendo a pesar del declive en el funcionamiento con la edad, y practican una reducción en su perspectiva temporal, así como un énfasis en el día presente, en el aquí y el ahora. Algunos estudios con participantes de 85 años muestran que se produce un retiro selectivo a un mundo interior, y un enfoque filosófico de la muerte como una transición hacia otra cosa. Otras investigaciones en la misma línea muestran en participantes de 80 años y más, un descenso en el autocentramiento y en el interés por las posesiones materiales, en hombres como en mujeres.

\section{Algo sobre pérdidas y longevidad}

A medida que se envejece se pierden capacidades y habilidades o destrezas que tal vez se tuvo en suerte desarrollar. Aumenta la lentitud, disminuyen las fuerzas, los pasos se acortan, la visión y oídos pierden agudeza. Existen variaciones importantes al respecto entre la población de As Ms, que tienen que ver con la genética y, muy especialmente, con el estilo más o menos saludable de vida llevado hasta ese momento. La longevidad aumentó a lo largo del siglo XX (y los más 
optimistas, en su delirio eufórico, señalaron que la ciencia y tecnología nos harían inmortales), e investigaciones publicadas en la revista $\mathrm{Na}$ ture muestran que la esperanza de vida máxima parece ser actualmente de 115 años, y la probabilidad de que haya una persona viva con 125 años, en cualquier lugar del mundo, es menos de 1 entre 10.000. Así, el límite de la longevidad no superaría 115 años e, interesantemente, esta edad es muy similar a aquella considerada como de la sabiduría e ideal en la tradición egipcia.

La longevidad no es necesariamente deseable, toda vez que se puede acompañar de una sumatoria de discapacidades y enfermedades que tornen la (calidad de) vida insoportable. No solo se pierden las capacidades de los órganos de los sentidos sino que, normalmente, se ha perdido al cónyuge - eventualmente los hijos-, a parientes y amigos, y, por tanto, el mundo parece cada vez más ajeno y distante. Así, frente al encarnizamiento terapéutico del postrado o enfermo terminal, cuando se quiere prolongar a toda costa la vida se debe tener la opción del rechazo y, como en países más desarrollados, avanzar rápidamente hacia la posibilidad legal y la viabilidad de la eutanasia, democratizada. Como siempre, la ciudadanía chilena parece más proclive a ésta que la superestructura de la sociedad y su "aparato" legal (uno de los más atrasados del mundo) y político. La discusión parlamentaria respecto de la eutanasia recién se ha iniciado en el parlamento chileno, y ojalá no se torne una tramitación eterna o ineficaz, como tantas otras iniciativas de real interés y necesidad para los ciudadanos ${ }^{3}$.

Respecto de la longevidad, un demógrafo y un médico detectaron que en la región de Barbaglia (Cerdeña, Italia) vivían más personas longevas que en otros lugares, y la marcaron en un mapa con color azul. Posteriormente se ha identificado cuatro zonas más: Okinawa (Japón), Icaria (Grecia), Loma Linda (California) y la Península de Nicoya (Costa Rica). Se trata de zonas altas, donde los habitantes se alimentan con

3 Paula Díaz, joven talquina (chilena) de 19 años, postrada víctima de una rara enfermedad degenerativa desde fines de 2013 y con dolores que le hacían insoportable vivir, envió video a la Pdta. M. Bachelet y posteriormente carta al Pdte. S. Piñera, suplicando morir. Su madre declaró que los médicos la habían abandonado (https://www.youtube. com/watch?v=6_VViKKDnYU). Murió, después de más de 5 años de sufrimientos, el 20 de enero de 2019 esperando una ley de eutanasia. 
no más de 1.800 calorías al día, principalmente de frutas y verduras ${ }^{4}$. En América Latina destaca, en Ecuador, Vilcabamba y su Valle Sagrado, a 50 kilómetros al sur de la ciudad de Loja (su ciudad más austral), y a 1.700 metros sobre el nivel del mar; es un entorno armonioso. Sus habitantes, aun después de los 100 años, mantienen sus facultades vitales intactas y realizan faenas agrícolas o ganaderas. Vilcabamba tiene un clima primaveral y contiene cañaverales y diversos árboles frutales, con temperaturas entre 18 y 22 grados todo el año. Su notoriedad proviene de la década de los setenta, cuando algunos científicos estudiaron por qué muchos habitantes de esa comunidad vivían más de 100 años, indicando que el clima, la apacible atmósfera, una sana alimentación con frutos de la tierra y, en especial, el agua de los ríos Chamba y Uchima y de los múltiples arroyos, serían los factores de la larga y saludable vida allí. Sus aguas contienen magnesio, hierro y otros minerales, por lo que consumirla propiciaría la quema de grasas y también ayudaría a prevenir el reumatismo.

Si bien la evidencia es a favor de llevar una alimentación y vida saludables (caminatas, alguna actividad física, evitar tabaco y exceso de carnes, etc.), también se observa la existencia de longevos no la han llevado así. Sorprendentemente, algunos pueblos de longevos consumen tabaco y ron, y en cantidades no menores. La esperanza de vida parece que tiene más que ver con genética y con haber vivido en ambientes incitantes al movimiento. Una gran mayoría de centenarios encuestados $(n=263)$ en las "zonas azules" pertenecían a una comunidad espiritual (cristianos, budistas, musulmanes, judíos o de otra religión). Aparentemente, pertenecer a una de estas comunidades aumenta la esperanza de vida. También, dan mucha importancia a la familia y viven rodeados de sus allegados. Los longevos han crecido además en círculos sociales "saludables", y el ser positivo y tener un compromiso de pareja puede añadir años a la esperanza de vida. Rodearse de buenos amigos contribuye en una gran medida a alargar la vida.

La encuesta británica Global Age Watch Index mide el bienestar de los ancianos en 96 países del mundo (que concentran el 91\% de los mayores de 60 años), en cuatro áreas: seguridad salarial, salud, capacidades personales y ambiente social favorable. De los 10 mejores países para vivir como A

4 El secreto de la longevidad en las "zonas azules": nueve razones para vivir más. The Conversation, July 11, 20186.49 pm EDT. Recuperado de https://theconversation.com/ el-secreto-de-la-longevidad-en-las-zonas-azules-nueve-razones-para-vivir-mas-99552 
M, siete son europeos (Noruega y Suecia los mejores), y de nuestra región latinoamericana el primero es Chile, y los últimos Venezuela y Honduras. Un alto Producto Interno Bruto (PIB) per cápita, sistemas de pensiones de amplia cobertura, buenos servicios de salud y ambientes sociales favorables son algunas de las cualidades de los primeros 20 países de la lista (BBC Mundo, 2014).

\section{Algunas conclusiones y sugerencias para futuros estudios}

Las relaciones sociales son muy importantes para el bienestar humano, y esto es particularmente relevante en últimas etapas del desarrollo. Un modo de estudiarlo, entre otros, es identificar y analizar el nivel de pertenencia de los As Ms a grupos sociales y sus consecuencias físicas y mentales, y distinguiendo sexos (Moyano-Díaz \& Mendoza-Llanos, 2021). Para facilitarlo, el Estado, muy lentamente, instala hogares diurnos (no superan aún los 20 en todo el país) para que los As Ms asistan a actividades apropiadas a su condición. Cualquiera sea la preferencia de participación social del A M debe ser aprovechada: conversar con viejos amigos o viejos conocidos, hacer nuevos contactos y nuevas amistades (superar el prejuicio de que los verdaderos amigos son solo los de la infancia o los antiguos), abrirse a nuevas relaciones y nuevos juegos contribuirá a mantenerse saludable.

Cada vez queda menos tiempo al A M y puede aprovecharlo bien, hacer lo que le gusta o le otorgue bienestar. Ante la certeza de la propia finitud y cercanía de la partida, es parte de un buen cierre del ciclo de vida, si se tiene bienes que legar a descendientes, dejar un testamento claro. También expresar a quienes están a su cuidado que, en caso de quedar grave, de muerte o imposibilitado de expresar voluntad, no haya encarnizamiento "terapéutico" que signifique prolongación de su vida innecesariamente, ya que éste frecuentemente significa empobrecimiento de la familia por los costos asociados: se puede afirmar la decisión de ser "desconectado" llegado el caso. Se puede luchar, cada vez que se pueda, para que los representantes al congreso aprueben la ley que permita acceso libre y democrático a la eutanasia; se busca evitar sufrir dolores insoportables que, frecuentemente, superan los "cuidados paliativos", y morir con dignidad. 
El A M tiene aún tiempo para dar gracias o para disculparse con quienes considere que lo merecen. Nunca es tarde para agradecer (ni para perdonar), ya que siempre hace bien al que lo hace, al que lo recibe y, así, a todos. Si el A M experimenta síntomas crecientes de olvidos, pérdida de memoria, confusión mental, desorientación espacial, irritabilidad, pérdida de actividades con sentido, problemas para mantener conversaciones y similares, debe consultar al médico geriatra o al gerontólogo, ya que la demencia es una enfermedad y NO es parte del desarrollo normal en la AM. Su aparición y su gravedad pueden ser retardadas con el aumento del consumo de legumbres, nueces, cereales, pescado y vitamina B6, todo lo cual ayuda a reducir el deterioro cognitivo y a prevenir la demencia.

Sería de alto interés que futuras investigaciones acerca de la psicología de los As Ms chilenos profundicen acerca de los estereotipos respecto de ellos, y particularmente el prejuicio y la discriminación en diferentes grupos sociales y profesionales, el efecto de la participación (o retiro) en grupos sociales, el impacto psicológico (además de material) de la jubilación y sus consecuencias según género, la fenomenología de la soledad, así como el efecto de la espiritualidad, creencias y religión en su bienestar y afrontamiento de la muerte. También, identificar si en nuestra cultura los As Ms viven las características de las dos últimas etapas según la teoría eriksoniana del desarrollo humano, las características de su desarrollo emocional mirado longitudinalmente y, particularmente, las propias de la nueva etapa denominada de la "gerotrascendencia", todo lo cual representa numerosos desafíos teóricos y empíricos de alto interés.

\section{Referencias}

Acerbi, C.N. (2013). Una mirada histórica. Y también la vejez tiene su historia. The old age has its history too. Revista de Salud Pública, (XVII)4: 69-73.

Alfaro, J. (2012). Posibilidades y tensiones en la relación entre Psicología Comunitaria y políticas sociales. En: J. Alfaro, A. Sánchez y A. Zambrano (Eds.), Psicología Comunitaria y Políticas Sociales. Reflexiones y experiencias (pp. 45-75). Buenos Aires: Paidós.

Alfaro, J. y Martín, M. (2015). Proceso y oportunidades de la transferencia del conocimiento desde la psicología comunitaria a las políticas públicas. Universitas Psychologica, 14(4), 1347-58. 
Alfaro, J. y Zambrano, A. (2009). Psicología Comunitaria y políticas sociales en Chile. Revista Psicología \& Sociedade, 21(2), 275282.

Alvarado, X. y Plaza, A. (2014). Bienestar subjetivo, calidad de vida y envejecimiento en Chile. En J.C. Oyanedel y C. Mella (Eds.), Debates sobre el bienestar y la felicidad (pp. 161-177). Santiago, Chile: RIL Editores.

Alvarado, X., Vargas, S., Reynaldos, K. y Oyanedel, J. C. (2016). Bienestar subjetivo y envejecimiento: una realidad en Chile entre los años 2011 y 2013. En E. Mendiburo, J. C. Oyanedel, D. Páez (Eds.). La felicidad de los chilenos (pp. 33-46). Santiago, Chile: USACH - RIL editores.

Ardila, R. (2003). Calidad de vida: una definición integradora. Revista Latinoamericana de Psicología, 35(2), 161-164.

Arias, C. (2013). Reflexiones finales acerca de la experiencia emocional en distintas etapas del ciclo vital. Revista Temática Kairós Gerontologia, 16(4), 205-215.

Arnold, M., Herrera, F., Massad, C. y Thumala, D. (2018). Quinta encuesta nacional de inclusión y exclusión social de las personas mayores en Chile: opiniones de la población chilena respecto al envejecimiento poblacional. Santiago, Chile: Ediciones Servicio Nacional del Adulto Mayor.

Arteaga, C. e Iñigo, I. (2015). Políticas sociales, modelo de desarrollo y subjetividad de grupos vulnerables en Chile. Revista del CLAD Reforma y Democracia, (61), 209-234.

Astete, M. y Vaccari, P. (2017). Políticas públicas y subjetividades: Lógicas en disputa en la implementación de programas sociales en la comuna de Lota, Chile. Psicoperspectivas, 16(1), 31-41.

Baltes P. B. \& Baltes, P. B. (1990). Psychological perspectives on successful (proactive) aging: Selective optimization with compensation. In P. B. Baltes \& M. M. Baltes (Eds.), Successful aging: Perspective from the behavioral sciences. New York: Cambridge University Press.

Berriel, F., Pica, C. y Zunino, N. (2017). Construcción social de la vejez en Uruguay a partir de documentos de políticas públicas. Psicoperspectivas, 16(1), 7-18. DOI 10.5927/Psicoperspectivas-vol16-issuel-fulltext-904.

Biblioteca del Congreso Nacional de Chile, BCN. (2002). Ley No 19.828: crea el Servicio Nacional del Adulto Mayor. Recuperado de 
https://www.leychile.cl/Navegar?idNorma=202950\&idVersi on $=2002-09-27$

Biblioteca del Congreso Nacional de Chile, BCN. (2011). Ley No 20.530: crea el Ministerio de Desarrollo Social y modifica cuerpos legales que indica. Recuperado de http://www.ministeriodesarrollosocial.gob.cl/btca/txtcompleto/mideplan_leg/ ley_20.530_midesocial.pdf

Brown, C. \& Lowis, M. J. (2003). Psychosocial development in the elderly: An investigation into Erikson's nine stage. Journal of Aging Studies, (17), 415-426.

Carmona, S. (2012). ¿Es la vejez lo que se dice de ella? Revista Temática Kairós Gerontologia, 15(4), 11-22.

Caro, S. (2014). Institucionalidad y Política Nacional para el adulto mayor en Chile: Análisis del período 1996-2012 desde una perspectiva institucionalista centrada en los actores. (Tesis de Máster). P. Universidad Católica de Chile, Santiago, Chile.

CASEN. (2017). Encuesta de Caracterización Socioeconómica. Adultos Mayores. Síntesis de resultados. Ministerio de Desarrollo Social, Observatorio social 2017. Recuperado de: www.desarrollosocial.cl el 9 de mayo de 2019.

Chackiel, J. (2004). La dinámica demográfica en América Latina. Serie Población y Desarrollo, $\mathrm{N}^{\circ} 52$. Santiago, Chile: Comisión para América Latina y el Caribe (CEPAL).

Cumming, E. \& Newell, W. (1961). Growing Old. The process of disengagement. $\mathrm{N}$ York: Basic Books.

Dávila León, O. (1998). Estado y políticas sociales: del Estado protector al Estado subsidiario. Última Década, (9), 1-9.

De Beauvoir, S. (1983). La Vejez. Edhasa.

Díaz, C. (2017). Migración internacional, envejecimiento poblacional y segunda transición demográfica, ¿hacia dónde va Chile? Notas de Población, (105), 221-257.

Erikson, E. (1974). Identidad, Juventud y Crisis. Buenos Aires: Editorial Paidós.

Erikson, E. (2000). El ciclo vital completado. Barcelona: Ediciones Paidós Ibérica. Versión extendida con nuevos capítulos del noveno estadio por Joan M. Erikson.

Fernández, L. M. B., González, R.S., Rosell, C. J. y Campos, C. T. (2016). Significados en torno a la jubilación y ser jubilado, de traba- 
jadores en edad prejubilación. Estudios sobre el adulto mayor. Santiago, Chile: Vice-rectoría Académica, U. Católica de Chile.

Fernández, F., Nazar, G. y Alcóver, C. M. (2018). Modelo de envejecimiento activo: causas indicadores y predictores en adultos mayores en Chile. Acción Psicológica, 15(2), 109-128.

Freudenberg, N. \& Tsui, E. (2014). Evidence, power, and policy change in community-based participatory research. American Journal of Public Health, 104(1), 11-14.

Fundación SOL. (2017). Pensiones: estudios predicen que medidas presidenciales no beneficiarán a la $3^{\mathrm{a}}$ edad. Radio Universidad de Chile, 29-05-2017. http://www.fundacionsol.cl/2017/05/ pensiones-estudios-predicen-medidas-presidenciales -nobeneficiaran-la-3a-edad/). Extraído 26-08-2019.

Ganga, F., Piñones, M., González, D. y Rebagliati, F. (2016). Rol del Estado frente al envejecimiento de la población: el caso de Chile. Convergencia. Revista de Ciencias Sociales, (71), 175-200.

Grijalva, G., Zúñiga, M. y Zupo, M. (2007). Adultas y adultos mayores en Sonora: ¿dependientes, autosuficientes o proveedores? Región y Sociedad, (19) (número especial), 117-145.

Huenchuan, S., González, D., Paredes, M. y Guzmán, J. (2007). Protección y participación en la vejez: escenarios futuros y políticas públicas para enfrentar el envejecimiento en Chile. Recuperado de http://www.senama.gob.cl/storage/docs/Protecciony-Participacion-en-la-Vejez-en-CELADE -CEPAL-2007.pdf

Lahera, E. (2008). Introducción a las Políticas Públicas. Santiago, Chile: FCE.

Lasagni, V., Bernal, R., Tuzzo, M., Rodríguez, M., Heredia, D., Muñoz, L., Palermo, N., Torrealba, L., Crespo, E., Gavira, G., Palacios, M., Villarroel, C., Fahmy, W., Charamelo, A., y Díaz, P. (2013). Estereotipos negativos hacia la vejez en personas mayores de Latinoamérica. Revista Kairós Gerontología, 16(4), 9-23.

Márquez-González, M., Izal, M., Montorio, I. y Pérez-Rojo, G. (2004). Emoción en la vejez: una revisión de la influencia de los factores emocionales sobre la calidad de la vida de las personas mayores. Rev Esp Geriatr Gerontol, 39(Supl 3): 44-51.

Márquez-González, M., Fernández de Trocóniz, M. I., Montorio Cerrato, I., Losada Baltar, A. (2008). Experiencia y regulación 
emocional a lo largo de la etapa adulta del ciclo vital: análisis comparativo en tres grupos de edad. Psicothema, 20(4), 616-622.

Martínez Ortega, M. P., Polo Luque, M. L. y Carrasco Fernández, B. (2002). Visión histórica del concepto de vejez desde la Edad Media. Cultura de los cuidados, VI(11), 40-46.

Ministerio de Planificación y Cooperación (MIDEPLAN). (1993). Política Nacional sobre Envejecimiento y Vejez: Lineamiento Básicos. Recuperado de http://www.ministeriodesarrollosocial.gob.cl/btca/txtcompleto/DIGITALIZADOS/Folletos\%20 Mide/mds-14-1993.pdf

Ministerio de Planificación y Cooperación (MIDEPLAN). (2000). Sistema integrado de evaluación de intervenciones públicas: Documento $N^{\circ} 4$, Metodología de análisis de políticas públicas, conceptos y criterios. Recuperado de http://www.ministeriodesarrollosocial.gob.cl/btca/txtcompleto/mideplan/mideplan_02_metodologia_analisis_polit_publicas.pdf.

Moyano, D. E. y Ramos, A. N. (2007) Bienestar subjetivo: midiendo satisfacción vital, felicidad y salud en población chilena de la Región Maule. Revista Universum, 2(22), 177-193.

Moyano, D. E., Núñez, D. y Jiménez, A. (2010). Calidad de Vida y Psicología. En E. Moyano D. (Ed.), Calidad de vida y Psicología en el Bicentenario de Chile. Imp. Marmor.

Moyano-Díaz, E. \& Mendoza-Llanos R. (2021). Membership, Neighborhood Social Identification, Well-Being, and Health for the Elderly in Chile. Front. Psychol, (11). 608482.

Moyano-Díaz, E., Mendoza-Llanos, R. y Páez, R. D. (2021). Psychological well-being and their relationship with different referents and sources of happiness in Chile. Revista de Psicología, 39(1), 161-182. DOI: https://doi.org/10.18800/psico.202101.007

Oerter, R. (1980). Moderna psicología del desarrollo. Ed. Herder.

Organización Mundial de la Salud (OMS). (1989). La salud de las personas de edad. Informe del comité de expertos de la OMS. Serie de informes técnicos 779. Ginebra, Suiza.

Organización Mundial de la Salud (OMS). (2016). Estrategia y plan de acción mundiales sobre el envejecimiento y la salud. 69a Asamblea Mundial de la Salud, Ginebra, 22 de abril del 2016 (A69/17). Recuperado de: http://apps.who.int/gb/ebwha/ pdf_files/WHA69/A69_17-sp.pdf 
Organización Panamericana de la Salud (OPS). (2017). Salud en las Américas. Edición del 2017. Resumen: panorama regional y perfiles de país. Washington DC, 2017.

Programa de Gobierno 2018-2022. Sebastián Piñera Echenique. Construyamos tiempos mejores para Chile. Recuperado de https:// www.sebastianpinera.cl/images/programa-SP.pdf

Quiroga, P., Albala, B. C. y Klaasen, G. (2004). Validación de un test de tamizaje para el diagnóstico de demencia asociada a edad, en Chile. Rev. Méd Chile, (132), 467-478.

Reyes, M., Olivares, B., Berroeta, H. y Winkler, M. (2015). Del discurso a las prácticas: Políticas sociales y psicología comunitaria en Chile. Polis, Revista Latinoamericana, 14(42), 387-413.

Roqué, M., Amaro, S., Massad, C., Herrera, F., Rovira, A., Karin, R y Ordano, S. (2016). Políticas Públicas sobre Envejecimiento en los Países del Cono Sur: Sistema Regional de Información y Aprendizaje para el Diseño de Políticas Públicas en torno al Envejecimiento. Recuperado de http://www.senama.gob.cl/ storage/docs/Politicas-publicas-vejez-cono-Sur.pdf.

Salvarezza, L. (1988). Psicogeriatría. Teoría y Clínica. Buenos Aires: Paidós.

Sánchez, X., Bailey, C. Arcos, E., Muñoz, L.A., González, L. \& Miranda, R. (2017). Subjective well-being and the perception of health opportunities: The case study of senior citizens of the neighborhood of Playa Ancha. World Development Perspectives, (5), 7-9.

Servicio Nacional del Adulto Mayor (SENAMA). (2004). Politica Nacional para el Adulto Mayor (Actualización).

Servicio Nacional del Adulto Mayor (SENAMA). (2012). Política Integral de Envejecimiento Positivo para Chile 2012-2025.

Servicio Nacional del Adulto Mayor (SENAMA). (2017a). Convención Interamericana sobre la Protección de los Derechos Humanos de la Personas Mayores. Recuperado el 20 agosto 2019 de http://www.senama.gob.cl/storage/docs/ Ratificacion-Conv-Interamericana-Prot-Derechos-PersMayores.pdf

Servicio Nacional del Adulto Mayor (SENAMA). (2017b). Informe de Seguimiento de Programas Sociales: Envejecimiento Activo. Recuperado de http://www.programassociales.cl/pdf/2018/ PRG2018_3_59434.pdf. 
Slachevsky, A. (2015). Descifrando la conducta humana desde el estudio del cerebro. Explora. Programa Nacional de Divulgación y Valoración de la Ciencia y la Tecnología. Recuperado el 20 de agosto 2019 de:

Sobieszczyk, T., Knodel, J. \& Chayovan, N. (2003). Gender and wellbeing among older people: evidence from Thailand (2003). Ageing and Society, (23), 701-735, Subdivisión: 6.

Stuardo, N., Zavala, M. y Merino, J. M.(2016). La decisión de jubilar. Dificultades y factores asociados en personal universitario de Concepción. Revista Perspectivas, (28), 79-107.

Thumala, D., Arnold, M., Massad, C. y Herrera, F. (2015). Inclusión y Exclusión social de las personas mayores en Chile. Santiago, Chile: SENAMA-FACSO U. de Chile. Ediciones Servicio Nacional del Adulto Mayor.

Tornstam, L. F. (2005). Gerotrascendencia: una teoría evolutiva del envejecimiento positivo. Nueva York: Springer Publishing Company.

Varela, L. (2016). Salud y calidad de vida en el adulto mayor. Revista Peruana de Medicina Experimental y Salud Pública, 33(2), 199-201.

Yuni, J. y Urbano, C. (2008). Envejecimiento y género: perspectivas teóricas y aproximaciones al envejecimiento femenino. Revista Argentina de Sociología, 6(10), 151-169. 